\title{
Diagnosi di polmonite atipica mediante multiplex PCR
}

\author{
Federico Piana, Alessandra Riccabone, Loredana Frisicale, Maria Quaranta, \\ Manuela Boetto, Giovanna Marchiaro, Daniela Maria Cirillo \\ UOA Microbiologia ASO San Giovanni Battista c.so Bramante 88 - 10126 Torino
}

Gli agenti eziologici più frequenti delle polmoniti atipiche comunitarie sono L.pneumophila, M.pneumoniae e C.pneumoniae. Questi ultimi due microrganismi causano patologie con identiche caratteristiche cliniche e radiologiche e diventa importante per il clinico avere una diagnosi rapida per instaurare una terapia adeguata.

L.pneumophila è l'agente eziologico della legionellosi, patologia in cui i sintomi della polmonite atipica sono accompagnati da una sintomatologia gastrointestinale, iponatremia e alterato stato mentale (2).

La diagnosi eziologica di polmonite sia nosocomiale sia comunitaria è utile solo se è effettuata in tempi rapidi e, possibilmente, su campioni ottenuti senza ricorrere a metodiche invasive. Le metodiche di recente introduzione che maggiormente rispondono a tali requisiti sono, oltre alla ricerca di antigeni mediante test immunocromatografici su secrezioni respiratorie o su urina, le indagini che utilizzano tecniche di amplificazione di geni in materiali provenienti dalle alte vie respiratorie. La Multiplex PCR (Pneumotris-Amplimedical Bioline) per L.pneumophila, M.pneumoniae e C.pneumoniae è un sistema rapido e di facile esecuzione che può essere usato anche su campioni raccolti senza l'uso di tecniche invasive.

La Multiplex PCR è una nested PCR che rivela la presenza del gene della citoadesina $\mathrm{P} 1$ di $M$. pneumoniae come un amplicone di 186 bp $(1,4)$ ed il gene OMP di C. pneumoniae (269 bp) (3). Per L. pneumophila il gene target è Mip (Macrophage Infectivity Potentiator). Il rilevamento dell'amplificato è eseguito su gel di agaroso $(2 \%)$ in presenza di etidio bromuro. Il controllo interno per la presenza di inibitori non specifici è eseguito sui campioni negativi amplificando il gene della $\beta$-globulina.

In questo studio retrospettivo abbiamo confrontato i risultati dell'amplificazione del DNA eseguita mediante multiplex PCR con quelli ottenuti con metodi tradizionali quali il rilevamento degli anticorpi IgM per M.pneumoniae e C.pneumoniae e la coltura per L. pneumophila. ̇̀ stata anche eseguita la ricerca dell'antigene urinario di Legionella. (Binax $\mathrm{NOW}^{\circledR}$ Legionella Urinary Antigen Test) $(5,6)$.

Nel periodo di tempo compreso tra maggio 2000 e febbraio 2003, abbiamo utilizzato la multiplex PCR per la rivelazione simultanea di amplificati specifici di L.pneumophila, M.pneumoniae e C.pneumoniae su 601 materiali (332 lavaggi broncoalveolari, 109 broncoaspirati, 20 liquidi pleurici, 37 biopsie respiratorie, 15 aspirati tracheali, 86 espettorati e 2 essudati/pus) su cui è stata richiesta la ricerca di almeno uno dei tre patogeni. I campioni sono stati opportunamente diluiti ed il DNA estratto tramite termolisi alcalina $\left(95^{\circ} \mathrm{C}\right.$ per 5 minuti). Gli estratti sono stati purificati per incubazione con Extracellular Purification kit ed i surnatanti sono stati raccolti ed amplificati secondo le istruzioni della ditta produttrice (Amplimedical).

53 campioni dei 601 testati $(8,8 \%)$ sono risultati positivi: 25 lavaggi broncoalveolari (8 per C.pneumoniae, 9 per L.pneumophila e 8 per M.pneumoniae); 11 broncoaspirati (8 per legionella e 3 per M.pneumoniae), 7 biopsie respiratorie ( 2 per M.pneumoniae, 1 per C.pneumoniae e 4 per L.pneumophila), 2 aspirati tracheali per L.pneumophila, 22 espettorati (7 per M.pneumoniae, 5 per C.pneumoniae, 1 per entrambe e 9 per L.pneumophila) ed 1 essudato/pus per L.pneumophila. Nessun liquido pleurico è risultato positivo per la ricerca di questi microrganismi.

Quando abbiamo confrontato questi risultati con quelli ottenuti mediante tecniche tradizionali, si è riscontrato che i 20 campioni positivi per M.pneumoniae sono stati raccolti da 19 pazienti. Da 10 di questi pazienti è stato raccolto un campione ematico per la ricerca delle IgM specifiche per questo microrganismo e in 2 casi la ricerca ha dato esito positivo $(20 \%)$.

Per quanto riguarda L.pneumophila, il campione è stato seminato su Charcoal Yeast Agar, ma le colonie sono cresciute in 14 casi sui 33 risultati positivi all'amplificazione $(42,4 \%)$. I 33 campioni provenivano da 18 pazienti e in 16 casi è stata eseguita anche la ricerca dell'antigene urinario: 2 sono risultati negativi e $14(87.5 \%)$ positivi. La ricerca delle IgG specifiche è stata richiesta per 16 pazienti e ha dato esito positivo in 10 casi $(62,5 \%)$.

In conclusione, i risultati ottenuti suggeriscono che se c'è un'ipotesi clinica di polmonite atipica, l'amplificazione contemporanea per la ricerca 
degli acidi nucleici di L.pneumophila, M.pneumoniae e C.pneumoniae permette di ottenere risultati diagnostici in una sola giornata lavorativa.

Il confronto tra l'amplificazione e la ricerca delle IgM specifiche per M.pneumoniae e C.pneumoniae evidenzia che un risultato positivo della prima correla maggiormente con la situazione clinica.

L'amplificazione di una regione conservata di L.pneumophila con primers specifici si è dimostrata un sistema veloce, specifico e sensibile per la diagnosi di legionellosi. Deve essere eseguita con il controllo dell'inibizione ed è complementare alla ricerca dell'antigene solubile nelle urine.

\section{BIBLIOGRAFIA.}

1. Abele-Horn et al. Molecular approaches to diagnosis of Pulmonary Disease due to M. pneumoniae. JCM $1998 ; 36,548-551$

2. Bernstein et al. (1998). Principles of Internal Medecine. McGraw-Hill

3. Boman et al.. Molecular diagnosis of Chlamydia pneumoniae Infection. JCM Dec 1999; 3791-3799.

4. Dorigo Zetsma et al. Molecular detection of M. pneumoniae in Adults with Community Acquired Pneumonia Requiring Hospitalization. JCM Mar 2001, 1184-1186.

5. Helbig J.H. et al. JCM Clinical Utility of Urinary Antigen Detection for Diagnosis of CommunityAacquired, Travel-Associated, and Nosocomial Legionnaires'Disease. JCM Feb. 2003. (41) 838-840.

6. Wever P. et al. Rapid diagnosis of Legionnaires' disease using an immunocromatographic assay for Legionella pneumophila serogroup 1 in urine during an outbreak in the Netherlands JCM 2000; 27382739 .

Daniela M. Cirillo

Ospedale San Raffaele

Emerging Bacterial Pathogens

Via Olgettina, 60 - 20132 Milano

Tel. 02-26437949

E-mail: cirillo.daniela@hsr.it 